\title{
Carcinoma del Cuello Uterino
}

\section{Revisión de 58 casos tratados Quirúrgicamente}

\author{
Por los Doctores Jorge Villarreal Mejía \\ y Eduardo Cáceres Alvarez*
}

El objeto de este trabajo es presentar los datos estadísticos obtenidos en la revisión de todos los casos de carcinoma de cuello uterino que han sido tratados en el Departamento de Ginecología del Hospital San Juan de Dios de Bogotá, Universidad Nacional de Colombia, en los últimos 5 años.

No se contemplarán aquí los detalles de las técnicas usadas ni se harán comentarios generales acerca del cáncer cervical por considerar que éstos han sido suficientemente descritos en la ex celente literatura mundial sobre el tema.

En la literatura médica colombiana se han publicado varias series de trabajos estadísticos sobre cáncer cervical tratado quirúrgicamente en un total aproximado de 200 casos. Consideramos que los aquí descritos constituyen una adición necesaria a la formación progresiva de nuestras propias estadísticas sobre tan importante tema.

\section{MATERIAL}

Comprende este estudio la revisión de 58 casos consecutivos de carcinoma de cuello uterino tratados quirúrgicamente; solamente en 53 se encontraron datos suficientes para la correcta clasificación clínica e histopatológica. De los otros cinco, tres casos no tenían diagnóstico clínico pre o postoperatorio, así como tampoco estudio histopatológico de la pieza quirúrgica. En los dos restantes, el diagnóstico clínico de invasión, pudo hacerse con base en el diagnóstico preoperatorio en el uno y en diagnóstico postoperatorio y estudio anatomopatológico en el otro.

La mayoría de las pacientes tratadas presentaban neoplasia de evolución reciente o sea estados I y II. No obstante por ra-

\footnotetext{
* Del Departamento de Ginecología del Hospital San Juan de Dios de Bogotá.
} 
zones de order hospitalario se operaron varios casos en estados avanzados en aquellas ocasiones en las cuales por el gran volumen de trabajo en el Instituto Nacional de Cancerología fue imposible asegurar a las enfermas la administración del tratamiento indicado en estos casos o sea la roengenoterapia y radiumterapia.

El personal científico del Servicio de Ginecología que actuó como equipo quirúrgico en los casos aquí presentados fue en su mayoría del nivel de los antiguos "Jefes de Clínica"; varios casos fueron intervenidos tambien por profesores del Servicio.

La técnica quirúrgica usada en la casi totalidad de los casos fue la preconizada y difundida por Wertheim-Taussig, con pequeñas variaciones personales impuestas por la diversidad de cirujanos que intervinieron en este grupo de pacientef

La disminución del número de casos por año, que se aprecia en el cuadro $\mathrm{N}^{0} 1$, debe explicarse por el mayor número de pacientes que han sido referidas últimamente al Instituto Nacional de Cancerología debido a sus crecientes facilidades para el tratamiento y admisión de pacientes carcinomatosas, así como al desarrollo de un criterio más estricto en la escogencia de los casos quirúrgicos adoptado en este Departamento en los últimos años. En ningún caso deberá interpretarse esa disminución aparente en el número de casos tratados en esta Institución, como una disminución en el volumen de pacientes con cáncer del cuello uterino.

\section{METODO}

Con el objeto de hacer fácilmente comparables los datos obtenidos en este grupo de pacientes con los de series similares tanto colombianas como extranjeras, se extrajeron de las historias estudiadas aquellos datos usualmente reportados de Edad, Paridad, Síntoma inicial, Exámenes preoperatorios, Complicaciones etc. En la parte final del trabajo se presentará un estudio estadístico de los resultados de supervivencia según el criterio recientemente enunciado por Butcher (2). 


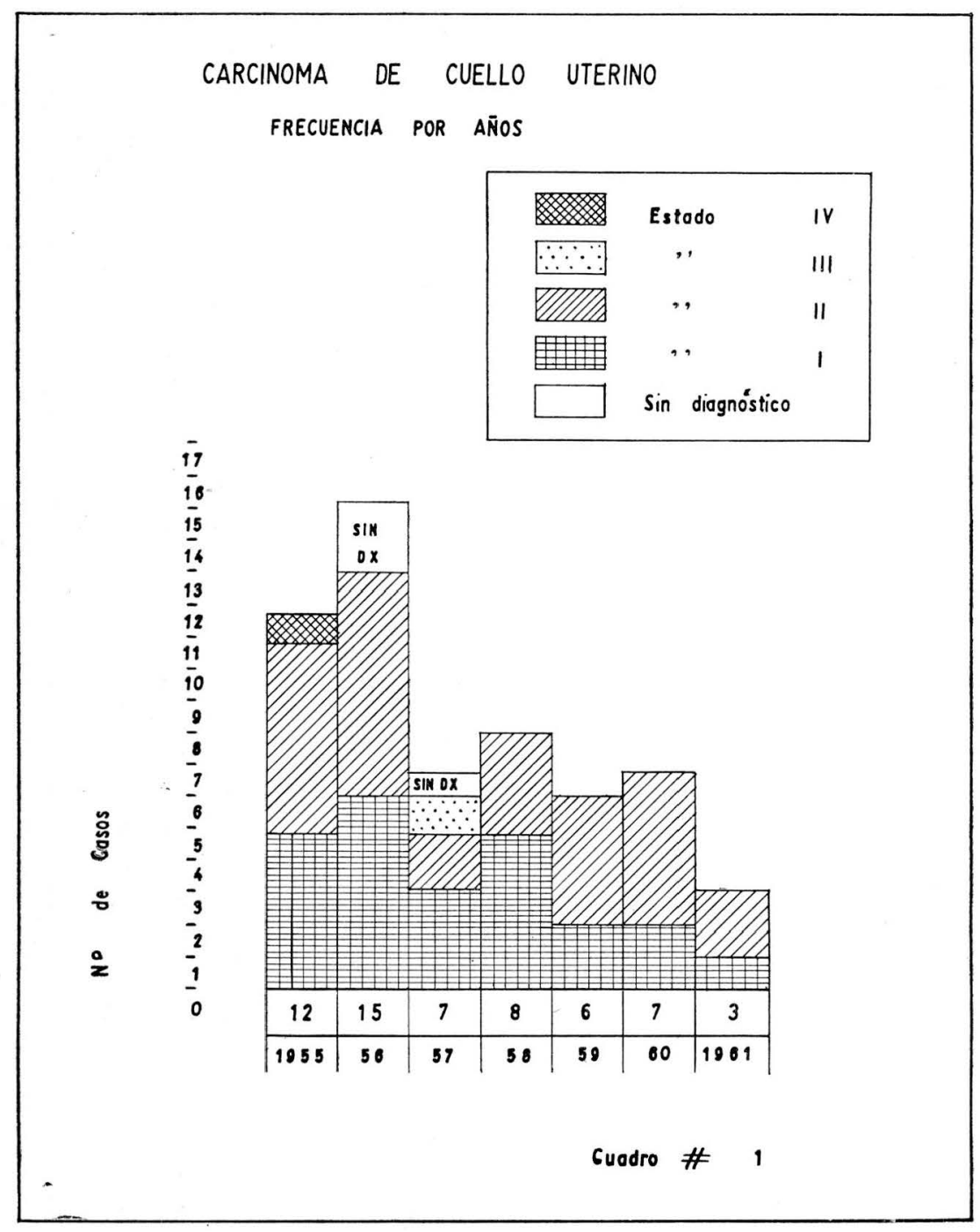

\section{RESULTADOS}

Edad:

La agrupación por edades en grupos de 5 años puede observarse en el cuadro $\mathrm{N}^{\circ}$ 2, apreciándose que el $44.8 \%$ de los casos estuvieron comprendidos entre los 40 y los 50 años de edad y un $29.3 \%$ por debajo de los 40 años. 


\section{AGRUPACION POR EDADES}

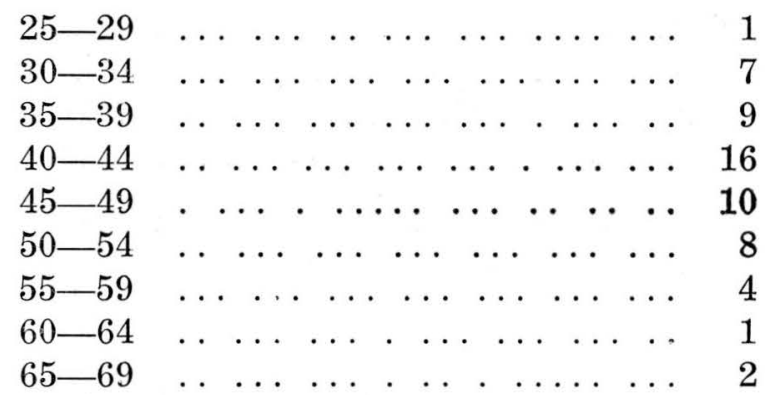

La paciente más joven fué de 26 años.

La paciente de más edad fué de 65 años.

\section{CUADRO No 2}

El promedio de edad para este grupo de pacientes fué de 43 años igual al que Welch y col. de la Mayo Clinic (22) encontraron entre 486 casos estudiados.

Estos datos nos ponen de presente una vez más el hecho de que en la actualidad el Cáncer cervical no debe considerarse como privativo de la $5^{\text {a }}$ década de la vida y que también entre nosotros el Cáncer debe buscarse aún en pacientes relativamente jóvenes.

\section{Paridad:}

Todas las pacientes en este estudio habían tenido hijos (cuadro $N^{0} 3$ ). Un $58.6 \%$ había tenido más de 6. El trabajo de Welch, mencionado anteriormente, revela un promedio de paridad de 2 partos por paciente y entre nosotros, López Escobar (12) reportó un $100 \%$ de multiparidad.

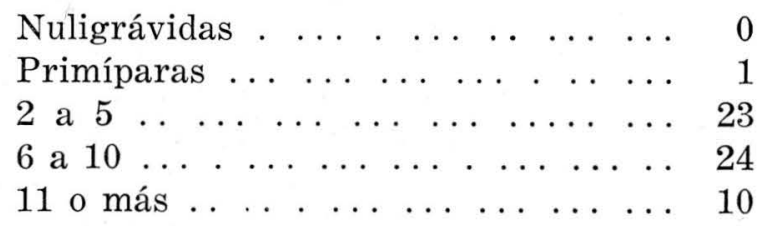

La paciente de mayor gravidez había tenido 15 hijos. 
Sintomatología:

Como síntoma inicial, fué hallazgo constante la presencia de hemorragia genital intermenstrual o postcoito en 43 de las 58 pacientes estudiadas. El flujo genital fétido siguió en importancia a la hemorragia como síntoma y causa principal de consulta.

El cuadro $N^{0} 4$ nos muestra la frecuencia de los diversos síntomas iniciales causa de consulta.

\section{SINTOMA INICIAL}

Hemorragia Genital: 43

Hemorragia postcoito, esfuerzo o ducha ........ . 11

Post-menopáusicas ... $\ldots \ldots . \ldots \ldots \ldots$

Hemorragias irregulares, intermenstruales . . . . 29

(Tipo metrorragias)

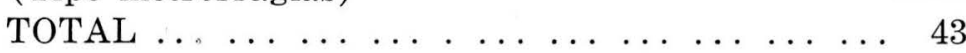

Dolor abdominal ... . . . . . . . . . . . . . . . . 2

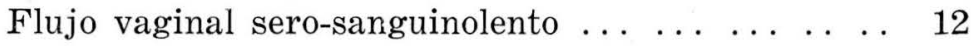

(4 pacientes más mencionaron el flujo como síntoma secundario)

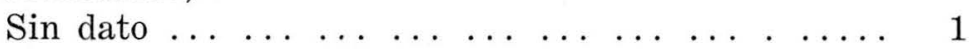

\section{CUADRO No 4}

\section{Demora en el Diagnóstico.}

Solamente en 51 de los 58 casos pudo hallarse dato al respecto. El promedio fue de 7.4 meses por paciente teniendo como criterio, el aceptado, de un mes o más, luego de iniciada la sintomatología causa de consulta. En 12 pacientes se encontró demora en el diagnóstico, inculpable al médico con un promedio de 3.7 meses para cada caso. El máximum de demora achacable al mético fue el de una enferma a la cual, 9 meses antes de diagnosticársele carcinoma se le practicó una histerectomía subtotal sin examen previo de cuello uterino por biopsia o citología. En 46 pacientes se encontró datc de demora achacable a la misma enferma con un promedio de 7.2 meses por caso. El de mayor demora fue un caso de una mujer de 40 años quien consultó por hemorragia postcoito y postesfuerzo de 4 años de duración. Como datos comparativos citamos los reportados por la Universidad 
de Wisconsin (9) de $21 \%$ y $67 \%$ de frecuencia para médico y paciente respectivamente y de 9 meses como promedio para cada uno.

Entre los errores diagnósticos más prominentes vale la pena destacar con fines puramente didácticos los siguientes: En paciente vista en la consulta externa un año antes dei diagnóstico no se practicó examen pélvico. A otra enferma con seis meses de sintomatología hemurrágica no se le practicó biopsia alguna para seis meses más tarde diagnosticársele un carcinoma estado II. Una paciente fue ooforectomizada un año antes del diagnóstico de carcinoma cervical. Al tiempo de la primera intervención presentaba, según la historia, una erosión cervical que no fue estudiada.

\section{Exámenes Preoperatorios:}

Un estudio preoperatorio adecuado de la paciente que va a intervenirse, como tratamiento para carcinoma cervical, requiere la ayuda de múltiples exámenes de laboratorio. Podemos dividir estos en dos tipos principales. El primero, aquellos exámenes llamados de rutina que nos van a dar una idea del estado general de la paciente, es decir: Cuadro Hemático, Examen de Orina, Azoemia, Glicemia, Tiempo de Coagulación. Es de anotarse la conveniencia de practicar también rutinariamente, previo a este tipo de cirugía, determinación de proteínas séricas y balance electrolítico de base.

El segundo tipo de pruebas de laboratorio estaría constituído por aquellos exámenes especiales destinados a descartar la posibilidad de metástasis lejanas o invasión de vecindad del cáncer cervical y entre estos los principales requeridos son: Cistoscopia, Rectosigmoidoscopia y estudio Radiográfico de pulmones y sistema urinario.

Dentro del grupo de exámenes de rutina, solamente a 32 pacientes en este estudio se les practicó la totalidad de exámenes previamente aconsejados como requerimiento mínimo. La anormalidad más frecuentemente encontrada en este grupo fue la de cifras bajas de hemoglobina (8 casos).

Solamente dos casos presentaron complicaciones operatorias o postoperatorias posiblemente relacionadas con cifras deficien- 
tes en los exámenes de laboratorio. Estos fueron: a) un caso de gran hematoma de cúpula en paciente con tiempo de coagulación prolongada y b) un caso de shock operatorio en una paciente anémica, con hemoglobina por debajo de $10 \mathrm{gms}$.

Veintiuna pacientes tenían determinación de proteínas, la mayoría dentro de límites normales. Así mismo, solamente 8 tuvieron determinación preoperatoria de base, de sus electrolitos, todas dentro de límites normales.

\section{Exámenes Especiales:}

Considerando como estudio mínimo preoperatorio para descartar metástasis e invasión a órganos vecinos los de Cistoscopia, Rectosigmoidoscopia y/o colon por enema así como Radiografía de tórax, encontramos que solamente 31 de los casos estudiados llenaron este requisito. Entre estos, 8 casos tenían además estudio Radiográfico de vías urinarias altas, el cual hoy día se considera obligatorio, pues además de aportar datos valiosos en cuanto a la invasión parametrial (evidenciada por estrecheces del extremo distal del uréter) este mismo dato tiene según Stander y col. (20) gran valor pronóstico. Estos autores, sobre un total de 189 pacientes, hallaron un $36 \%$ de supervivencia superior a un año en aquellas enfermas cuyas urografías pretratamiento eran normales en comparación con las enfermas que presentaban imágenes anormales con evidencia de estrechez ureteral. El dato discriminado de exámenes especiales tomados a las pacientes en estudio puede observarse en el cuadro $\mathrm{N}^{0} 5$ siendo de anotarse el bajo número de estudios pulmonares.

\section{EXAME'NES ESPECIALES}

Biopsia positiva para Carcinoma: en 48 casos Sin Biopsia en la historia: 10 casos Radiografía de Tórax: Negativa: $\quad 39$ casos Cistoscopia: Negativa: 47 casos

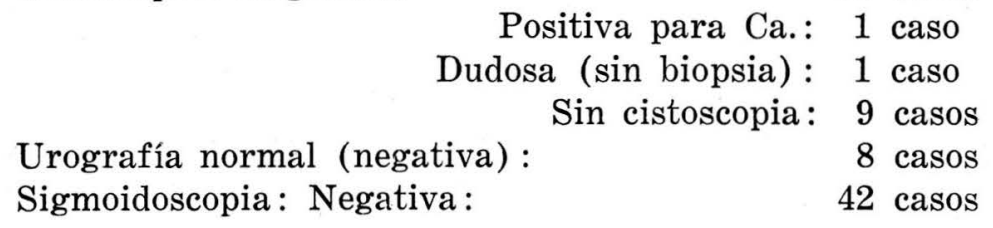


Proctitis amibiana: 3 casos

Mucosa sana tabique RV invadido: 1 caso

Colon por enema, negativo: 1 caso

Columna vertebral negativa: $\quad 3$ casos

Abdomen simple, negativo: 2 casos

Sin ningún estudio radiográfico: 13 casos

Fstado cardiovascular bueno: 13 casos

Estudio Cardiovascular:

14 casos

Malo pero sin contraindicación cirugía: 1 caso

\section{CUADRO N 5}

Considerando que la cirugía para cáncer cervical representa de por sí un trauma bastante grande dados el tiempo de anestesia, la extensa disección visceral y la pérdida de sangre usualmente grande; en los últimos 14 casos operados en el Departamento de Ginecología de San Juan de Dios hemos usado como estudio adicional preoperatorio el concepto cardiovascular sobre riesgo quirúrgico siendo en todos ellos satisfactorio. Ninguna de estas pacientes presentó complicaciones operatorias o en el postoperatorio inmediato.

\section{Estados Clínicos:}

El diagnóstico clínico de los casos revisados se llevó a cabo de acuerdo con el criterio aceptado de clasificación internacional conocido también con el nombre de Clasificación de la Liga de las Naciones. Debido a lo incompleto de algunas historias en este estudio, no fue posible clasificar desde este punto de vista a tres de las pacientes. No 6.

La distribución por casos puede apreciarse en el cuadro

\section{ESTADOS CLINICOS}

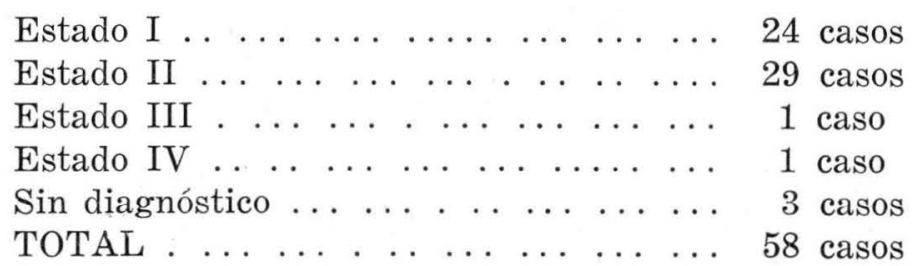

CUADRO No 6 
El error en la clasificación del carcinoma de cuello uterino por estados, teniendo como base la apreciación clínica, es de frecuente ocurrencia y para diferentes autores (19-7) fluctúa entre el 16 y $24 \%$. En esta serie de casos, 10 tuvieron un diagnóstico postoperatorio menos grave que el preoperatorio, entre tanto que 14 presentaban un grado de invasión o extensión mayor que el encontrado clínicamente. El detalle de estas variaciones en los diagnósticos puede apreciarse en el cuadro siguiente:

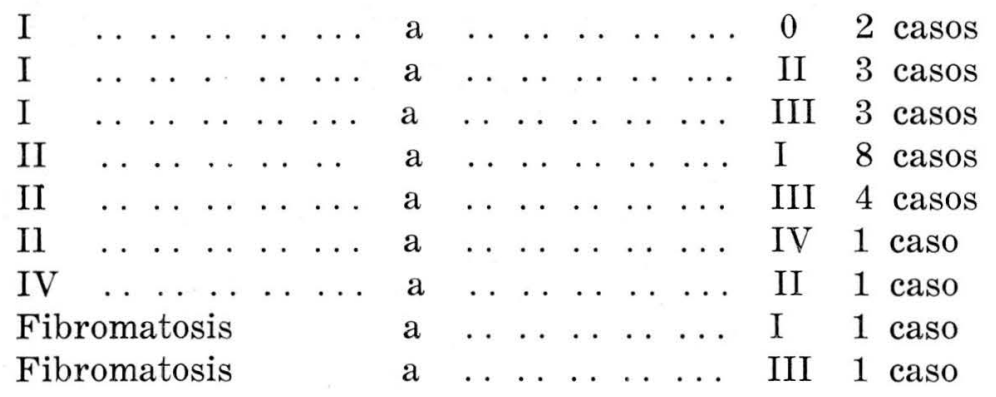

CUADRO No 7

Tipo de Cirugía:

La agrupación de los casos según el método quirúrgico empleado puede observarse en el cuadro $N^{0} 8$.

\section{TECNICAS QUIRURGICAS}

Histerectomía radical ... . . . . . . . . . . . 50 casos

Schauta ... . . . . . . . . . . . . . . . . . . 1 caso

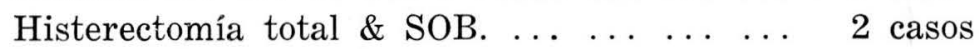

Histerectomía subtotai \& S. O. D. ... . . . 1 caso

Histerectomía vaginal amplia Salp. B. . . . 1 caso

Linfadenectomía \& SOB. ... . . . . . . . . . . . 1 caso

Ext. cuello restante. Linfd. pel. ... . . . ... 1 caso

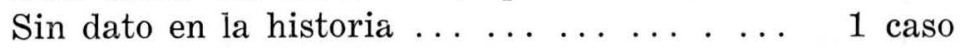

\section{CUADRO No 8}

* La Histerectomía tipo Schauta fué practicada en una paciente de 40 años con un prolapso genital de III grado, carcinoma estado I, quien a los 3 años de postoperatorio presentaba una pelvis enteramente negativa. 
* Las pacientes con histerectomía total y SOB llegaron a Cirugía con diagnósticos errados. Una fue intervenida con diagnóstico de Carcinoma de endometrio encontrándose posteriormente un Ca de cuello estado II. La supervivencia en esta paciente fue de un año solamente. La segunda enferma en este pequeño grupo fue operada con diagnóstico de Fibromatosis uterina. En la laparotomía sospecharon el cáncer cervical, pidieron estudio histológico por congelación e! cual confirmó el cáncer, pero la intervención no pudo ser terminada por mal estado de la paciente. Luego de diagnóstico de Ca estado I, la paciente recibió roentgenoterapia en forma incompleta y ha tenido una supervivencia de 4 años y medio.

* El caso intervenido con histerectomía subtotal fue el de una enferma de 45 años a quien con diagnóstico de fibromatosis uterina e historia de hemorragias irregulares durante 12 meses, no se le practicó biopsia de cuello previa a la operación planeada como histerectomía total. En la intervención se encontró invasión pélvica generalizada diagnosticada como Ca estado III. La enferma recibió roengenoterapia profunda en el Instituto Nacional de Cancerología y se encontraba viva en el último control, a los dos años de operada

* El siguiente caso en este breve resumen de las pacientes a las cuales no se les practicó histerectomía radical, corresponde a una enferma de 65 años con prolapso genital de III grado a quien se le practicó una "Histerectomía Vaginal ampliada" con salpingectomía bilateral y resección de $3 / 4$ partes de vagina. Presentó absceso de cúpula y necrosis de la herida. El diagnóstico quirúrgico fue de estado II. En el postoperatorio recibió una dosis de $3.300 \mathrm{R}$ en tumor y murió a los 4 meses del postoperatorio luego de haber abandonado el tratamiento.

* En un caso se practicó linfadenectomía con SOP en una paciente con diagnóstico clínico de $\mathrm{Ca}$ estado II a quien en el acto quirúrgico se le encontró una gran invasión pélvica, motivo por el cual no se extirpó el útero. No se le pudo administrar radiumterapia ni roengenterapia perdiéndose de vista a los dos meses del postoperatorio.

* Por último, en una naciente de 50 años a quien tres meses antes se le había practicado una histerectomía subtotal sin estu- 
dio histológico de cuello, se le hizo una extirpación de cuello restante y linfadenectomía pélvica con diagnóstico de Carcinoma estado II. Los cuatro meses de control no han revelado metástasis alguna.

Cuatro de los seis casos que recibieron tratamiento quirúrgico incompleto murieron en el postoperatorio próximo o fueron perdidos de vista. Es de anotar sin embargo la supervivencia relativamente larga, de 4 a 2 años respectivamente, en dos casos incompletamente tratados.

\section{Hallazgos Quirúrgicos:}

En el año de 1952, Meigs y Brunschwig (14) propusieron una nueva clasificación del carcinoma de cuello uterino basada en los hallazgos quirúrgicos (Véase cuadro $\mathrm{N}^{\circ} 9$ ). La importancia pronóstica de esta clasificación se fundamenta en el hecho que dentro de los estados II según la clasificación internacional, aquellos casos que presentan una invasión o extensión tumoral en sentido vertical, es decir vagina o cuerpo uterino, conllevan generalmente una mayor supervivencia que el grupo restante de estados II en el cual la propagación o invasión tumoral se hace en sentido lateral, para-cervical o para-vaginal. Según la clasificación propuesta por dichos autores, el primer grupo mencionado estaría incluído en el estado $\mathrm{B}$ y el segundo en los grupos C y D. Las cifras de supervivencia reportadas por los proponentes de esta nueva clasificación para los diferentes estados, fueron de $82 \%$ para el estado B. $50 \%$ para el C y de $37 \%$ para el D. Creemos con otros autores que la marcada diferencia de supervivencia en grupos diversos dentro del mismo estado II de la clasificación internacional, justifica ampliamente, sobre todo desde el punto de vista de su valor pronóstico, el uso de esta nueva clasificación.

En el cuadro $\mathrm{N}^{\circ} 9$ podemos apreciar la distribución de los casos presentados en esta serie, según los diferentes estados para ambos tipos de clasificaciones, la Internacional o de la Liga de las Naciones y la aquí enunciada de Meigs y Brunschwig. El pequeño número de casos controlados al término de cinco años, nos abstuvo de intentar la presentación de cifras de supervivencia comparables a las mencionadas previamente por los autores extranjeros. 
HALLAZGOS QUIRURGICOS

\begin{tabular}{|c|c|c|c|}
\hline $\begin{array}{l}\text { Clasific. } \\
\text { Meigs } \\
\text { Brunschwig }\end{array}$ & Extensión del Carcinoma & $\begin{array}{l}\text { Clasific. } \\
\text { Inter. }\end{array}$ & $\begin{array}{l}\text { Ne Casos } \\
\text { H.S.J.D.D. }\end{array}$ \\
\hline A & Lesión circunscrita a cuello & & 28 \\
\hline A 0 & $\begin{array}{l}\text { Carcinoma invasivo en la biopsia ini- } \\
\text { cial que no se encuentra en pieza }\end{array}$ & & 2 \\
\hline B & $\begin{array}{l}\text { Extensión a cuerpo Uterino o dos ter- } \\
\text { cios sup. de Vag. }\end{array}$ & & $9 *$ \\
\hline $\mathrm{C}$ & $\begin{array}{l}\text { Extensión a áreas pericerv. y periva- } \\
\text { ginales y } 1 / 3 \text { inferior de vagina. }\end{array}$ & & $\begin{array}{l}12 \\
* *\end{array}$ \\
\hline D & $\begin{array}{l}\text { Extensión a ganglios linfáticos de la } \\
\text { pelvis, fuera de } C \text {. }\end{array}$ & & $\begin{array}{l}3 \\
* * *\end{array}$ \\
\hline$E$ & Extensión a vejiga o Recto & & 2 \\
\hline $\mathrm{F}$ & $\begin{array}{l}\text { Extensión a pared pélvica o metástısis } \\
\text { distant. }\end{array}$ & & \\
\hline
\end{tabular}

\section{CUADRO No 9}

De los 9 casos* clasificados según Meigs y Brunschwig como estado $\mathrm{B}$, solamente dos presentaron recidiva de la lesión, 16 y 19 meses después de la operación. Por otra parte, entre los 15 casos en estado $\mathrm{C}^{* *}$ y $\mathrm{D}^{* * *}$, nueve presentaron recidivas tumorales en períodos de tiempo que fluctuaron entre dos y sesenta meses del postoperatorio El número de pacientes perdidas de vista en el postoperatorio inmediato fue de dos para B y C y de una para el estado D.

Anestesia:

La anestesia usada en la mayoría de los casos fue la general. Cuadro № 10. 


\section{ANESTESIA}

Anestesia general . . . . . . . . . . . . . . . . . 44 casos

Anestesia raquídea ... . ............ . . 12 casos

Anestesia peridural ... ... . ......... 1 caso

Anestesia raquídea + general ......... . 2 casos

Sin dato en Historia ... ............. . . 2 casos

\section{CUADRO N⿳0 10}

Un total de 41 pacientes recibieron un promedio de 931 c.c. de sangre total durante la intervención.

\section{Accidentes Operatorios:}

Catorce pacientes o sea el $24 \%$ presentaron accidentes operatorios. El accidente más frecuente fue la apertura de vejiga ocurrida en 7 casos. No se presentó ninguna muerte en la sala de cirugía (Cuadro No 11). Otros autores (19) reportan de 12 a $17 \%$ de complicaciones quirúrgicas.

\section{ACCIDENTES OPERATORIOS}

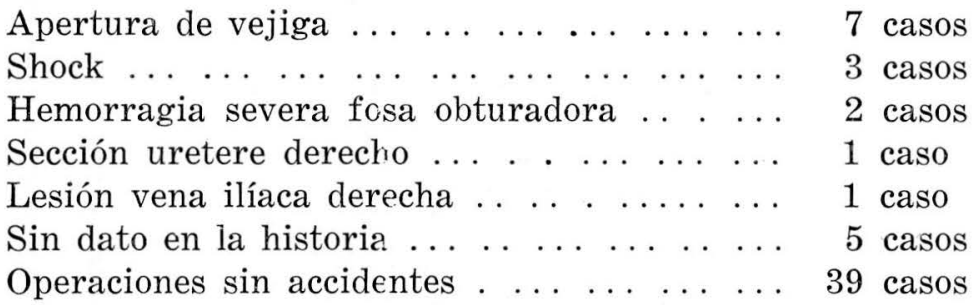

\section{CUADRO No 11}

Pese al pequeño número de casos presentados en este estudio, la mortalidad operatoria debe considerarse como baja.

\section{Complicaciones Postoperatorias:}

La complicación postoperatoria más frecuente en cirugía radical para carcinoma de cuello uterino es en la gran mayoría de los casos la aparición de fístulas urinarias. La incidencia de és- 
tas, según diferentes autores varía entre el 1\% (18) y el $23 \%$ (6-16-21). El concepto de Brunschwig es todavía respetado cuando dice (3) "es nuestra opinión que cuando la incidencia de estas complicaciones - fístulas del tracto urinario- es despreciable en eualquier serie significativa de pacientes, las operaciones hechas no han sido tan extensas y completas como ellas deben ser para el Carcinoma de cuello".

Las fístulas urinarias consecutivas a trauma quirúrgico directo son de rara ocurrencia. La gran mayoría de ellas se presentan a causa de la necrosis por falta de irrigación en la porción distal del uretere el cual. al ser disecado de los tejidos parametriales que lo rodean en el tiempo quirúrgico más importante de la histerectomía radical, va a quedar desprovisto de sus principales arterias nutricias. Una vez iniciada la necrosis del uretere y la infección con frecuencia presente en el espacio retroperitoneal, alrededor del $7^{\circ}$ al $10^{\circ}$ día se inicia la extravasación de orina que solamente entre el décimo cuarto y vigésimo primer día va a hacerse presente en forma de incontinencia urinaria permanente a través de la vagina $(6)$.

Como puede observarse en el cuadro $\mathrm{N}^{\circ} 12$ de complicaciones postoperatorias, la incidencia de fístulas urinarias en este grupo de pacientes fue de 14 en los 58 casos o sea el $24 \%$.

Entre nosotros y sobre un total de 90 casos López Escobar (12) reportó una incidencia del $15.5 \%$.

En la literatura reciente sobre carcinoma de cuello tratado quirúrgicamente, vale la pena destacar el trabajo de Novak (18), en Yugoeslavia quien preconiza el evitar la denudación circular del uréter en su extremo distal así como el que se deje intraperitonealmente. Con esta técnica y sobre un total de 100 intervenciones en los años de 1954 a 1956, solamente encontró una fístula.

Una nueva adición técnica en la búsqueda continua para reducir el número de fístulas postoperatorias, es la propuesta por Symmonds y Pratt (21) quienes aconsejan la succión continua del espacio retroperitoneal por 3 a 6 días. Este autor sobre un total de 20 casos no encontró ninguna fístula.

Desde el punto de vista diagnóstico vale la pena hacer hincapié aquí sobre el valor que últimamente se le está dando al estudio radiológico de vías urinarias no solo como pronóstico, men- 
cionado anteriormente, sirio también como diagnóstico precoz de las complicaciones urológicas de tipo fistuloso (1).

Mallik (13) anota que la hidronefrosis e hidrouréter son un hallazgo normal en todos los casos de cirugía radical como efecto del edema ureteral, el cual es evidente en las radiografías entre la 3-4 semanas para luego desaparecer espontáneamente. Aconseja tomar urografías excretoras tempranas al comienzo de la segunda semana postoperatoria las cuales, si revelaren hidrouréter, serían indicativas del probable desarrollo de fístulas urinarias.

\section{COMPLICACIONES POSTOPERATORIAS}

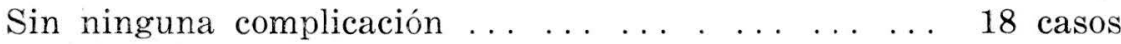
Historias incompletas ................... . . . . 5 casos

Infecciosas: (22 casos)

Absceso de cúpula vaginal . .......... 5 casos

Absceso de pared abdominal ... ... . . ... 9 casos

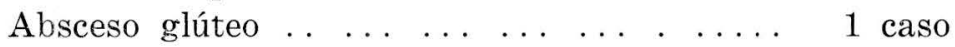

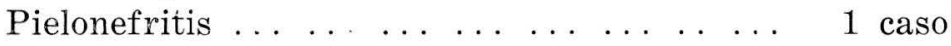

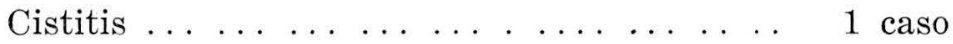

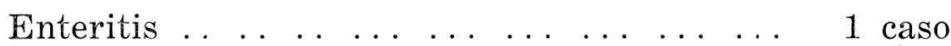

Flebitis . .................... . . . 2 casos

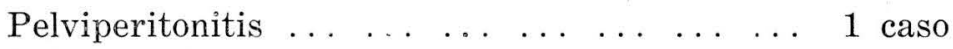

Bronconeumonía ................... 1 caso

Fístulas: (14 casos)

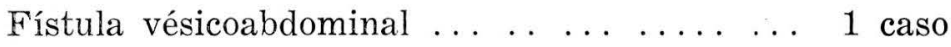

Fístula ureterovaginal . . ... ... ... . . . 6 casos

Fístula vésico-vaginal . . . . . . . . . . . . . . 6 casos

Fístula urétero-vésico-vaginal . . . . . . . . 1 caso

Hemorrágicas: (8 casos)

Hemorragia de cúpula vaginal . . . . . . . . 1 caso

Hemorragia abdominai, retroperitoneal ..... 2 casos

Hematoma de cúpula . . . . . . . . . . . . 2 casos

Hematoma de herida abdominal ... . ... . 3 casos 
Otras:

\begin{tabular}{|c|c|}
\hline & \\
\hline $\begin{array}{lllll}\ldots & \ldots & \ldots & \ldots\end{array}$ & \\
\hline intestinal $\ldots \ldots \ldots$ & \\
\hline ilibrio electrolítico severo . ... & \\
\hline $\begin{array}{llllllll} & \ldots & \ldots & \ldots & \ldots & \ldots & \ldots & \ldots\end{array}$ & \\
\hline . & \\
\hline$\ldots \ldots \ldots \ldots$ & \\
\hline identalmente en vagina . . & \\
\hline & \\
\hline & \\
\hline
\end{tabular}

Considerando las complicaciones postoperatorias en general encontramos una muy alta incidencia en ei grupo de casos estudiados por nosotros, $68.98 \%$.

El porcentaje de morbilidad informado por autores extranjeros en series similares ha sido del $20 \%$ (4), del $32 \%$ (19) y para el Instituto Nacional de Cancerología en Bogotá de 49\%. (8)

El número de complicaciones de tipo infeccioso fue de 22 en los casos aquí presentados o sea una incidencia del $37.9 \%$ entre tanto que Werthein y Meigs citados por Frick (5), Parsons y Friedel (19) encontraron para este tipo de morbilidad cifras respectivas de $17,4.9$ y $13.7 \%$.

La morbilidad febril, tomando como criterio el aceptado universalmente de morbilidad obstétrica, se presentó en 14 de las 58 pacientes, con un promedio de 4.8 días por paciente y un porcentaje general del $24.1 \%$.

\section{Mortalidad:}

El número de muertes ocurridas en la serie de casos aquí presentados y sometidos a control fue de siete en total. De éstas, dos ocurrieron en el postoperatorio inmediato, siete y nueve días respectivamente, siendo este el único dato que podemos presentar en forma absolutamente exacta y completa. Así pues la cifra de mortalidad operatoria y postoperatoria inmediata es de $3.4 \%$. En los 90 casos informados por López Escobar, la mortalidad 
postoperatoria fue de $5.5 \%$. Autores extranjeros, informando sobre series numéricamente superiores dan las siguientes cifras de mortalidad.

Parsons y colaboradores (19) 104 casos $0.9 \%$.

Frick y colaboradores (5) 117 casos $3.4 \%$.

Welch y colaboradores (22) 486 casos $1.9 \%$.

Incluímos a continuación un breve resumen de las siete muertes ocurridas en el presente estudio.

\section{HISTOKIA CLINICA No $113 \%$}

Paciente de 57 años de edad a quien se le practicó en 1955 una Histerectomía total con salpingooforectomía bilateral con diagnóstico preoperatorio de Carcinoma de Endometrio. El diagnóstico operatorio fue de Carcinoma de cuello uterino estado II comprohado en la pieza quirúrgica. Muere un año más tarde y a la autopsia se encuentra compresión ureteral bilateral, hidronefrosis bilateral, uremia, ascitis, congestión y edema pulmonar así como metástasis a ganglios linfáticos preaórticos.

\section{HISTOFIA CLINICA $\mathbf{N}^{0} 78816$}

Paciente de 50 años de edad En el año de 1955 se operó de un quiste ovárico en el Hospital de San Juan de Dios. En esa época presentaba según la historia una erosión cervical que no fue investigada. Un año más tarde consultó por flujo vaginal serosanguinolento de un mes de duración. Aparece en la Historia Clínica que fue intervenida con Histerectomía Radical, pero no existe dato acerca del estado cínico del tumor. Como complicación postoperatoria presentó una bronconeumonía de la que se recuperó. Volvió tres y medio años más tarde cuando se le diagnosticaron metástasis ganglionares en cuello. No se hizo tratamiento y muere a los 43 meses de operada.

\section{HISTORIA CLINICA No 61898}

Paciente de 30 años de edad. Con diagnóstico de carcinoma estado II, se le practica Histerectomía Radical a los dos meses de iniciada la sintomatología hemorrágica causa de consulta. En el acto quirúrgico presenta hemorragia severa en fosa obturadora y en el postoperatorio fístula urétero-vaginal bilateral, desequilibrio electrolííico severo y oclusión intestinal. El diagnóstico quirúrgico es de un estado III encontrándose ganglios ilíacos positivos para carcinoma. Dos meses más tarde se le practica urétero-neocistostomía y seis meses después de la cirugía rađical debido a persistencia de la fístula, se interviene de nuevo de Ileo-urétero-cistoplastia. Muere un mes más tarde luego de severo desequilibrio electrolítico e insuficiencia renal. 


\section{HISTORIA CLINICA No 185981}

Paciente de 65 años de edad. Luego de 12 meses con sintomatología de flujo piosanguinolento por vía vaginal, con diagnóstico clínico de Carcinoma estado I, y prolapso genital grado III, se le practica en 1959 una Histerectomía Vaginal amplia que incluye $3 / 4$ partes de vagina y Salpingooforectomía bilateral. El diagnóstico postoperatorio revela invasión a la vagina. En el postoperatorio inmediato presenta un absceso de cúpula y necrosis de los bordes de la herida. Además de diarrea severa y retención urinaria. Vista dos meses más tarde en el Instituto Nacional de Cancerología se le encuentra inva. sión parametrial. Recibe parte inicial de Roengenoterapia (3.300 r), abandona el tratamiento y muere 4 meses después de operada de causa desconocida.

\section{HISTORIA CLINICA No 198206}

Enferma de 35 años de edad, quien consulta luego de 7 meses de anorexia y flujo genital como únicos síntomas. Con diagnóstico de Carcinoma estado II parametrio izquierdo se le practica Histerectomía radical presentando hemorragia en fosa obturadora. Los hallazgos operatorios confirman el diagnóstico clínico. En el postoperatorio presenta pelviperitonitis, íleo paralítico y gran desequilibrio electrolítico muriendo anúrica en el $7^{\circ}$ día postoperatorio.

\section{HISTOEIA CLINICA No $1693 \% 0$}

Paciente de 57 años de edad. Con diagnóstico de Carcinoma cervical estado II (parametrio izquierdo) se le practica una Histerectomía radical durante la cual se abre vejiga. Los hallazgos operatorios e Histopatológicos en la pieza extirpada revelan lesión limitada al cuello uterino. En el postoperatorio presenta íleo paralítico, hipopotasemia e insuficiencia cardíaca. Muere en el

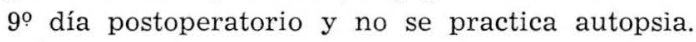

\section{HISTORIA CLINICA No $3 \% 127$}

Paciente de 38 años de edaci, quien consulta luego de 12 meses de estar presentando hemorragia abundante postcoito. Con el diagnóstico de carcinoma estado I, se le practica una Histerectomía radical confirmándose la impresión clínica preoperatoria. Presenta absceso de pared abdominal y tromboflebitis de las cuales se recupera. Segundo control a los tres meses revela invasión parametrial derecha por lo cual se envía al Instituto Nacional de Cancerología para radioterapia. Aparentemente la paciente no sigue las instrucciones dadas y a los 10 meses de opelada muere con carcinomatosis abdominopélvica generalizada.

\section{ANATOMIA PATOLOGICA}

Los estudios anatomopatológicos efectuados en los casos aquí presentados deben considerarse como insuficientes o inadecuados. Entre tanto que 10 casos no tuvieron biopsia cervical previa al tratamiento quirúrgico, el estudio histopatológico postoperatorio 
no se encontró en la historia en cuatro de los casos intervenidos. Es de suponer que prácticamente la totalidad de estas fallas han sido únicamente en el manejo de las historias clínicas pues es absurdo el suponer que se hayan intervenido enfermas de histerectomía radical para cáncer cervical. con base únicamente en impresiones clínicas. La clasificación de los casos según la interpretación de su grado histológico de malignidad no fue llevada a cabo en la mayoría de los casos por el departamento de patología y por este motivo no se menciona en este trabajo.

La importancia del estudio de las metástasis ganglionares ha sido demostrada por diferentes autores (11-15-19) sobre todo en cuanto respecta a su valor pronóstico sobre la supervivencia postoperatoria. Parsons (19) en un total de 104 casos reporta una frecuencia total de metástasis ganglionares del $19 \%$ y anota que la frecuencia de éstas no aumentó en los estados III y IV, en relación con la encontrada en los estados I y II. La supervivencia comparativa para aquellos casos con y sin metástasis ganglionares fueron de $50 \%$ y $78 \%$ para el estado I y de $20 \%$ a $68 \%$ para el estado II. Este mismo autor menciona los ganglios de la cadena obturadora e ilíaca común como los más frecuentemente afectados. También Meigs (15) sobre un total de 131 casos (estados I - II y III) operados entre 1940 y 1949 da una supervivencia total de $73 \%$. De estos 131 casos, 33 tenían ganglios positivos y dieron una supervivencia del $36 \%$ únicamente.

En los casos revisados por nosotros, solamente en 39 se encontraron ganglios en la pieza quirúrgica. Su distribución de acuerdo con positividad o negatividad así como en relación con el diagnóstico preoperatorio puede observarse en el cuadro № 13.

\section{GANGLIOS LINFATICOS}

10 pacientes con ganglios positivos para carcinoma

Diagnóstico preoperatorio: I . . . . . . . . . . . . 3 casos

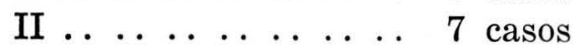

29 pacientes con ganglios negativos:

Diagnóstico preoperatorio: I . . . . . . . . . . 11 casos

II $\ldots \begin{array}{lllllll} & \ldots & \ldots & \ldots & \ldots & \ldots & 18 \text { casos }\end{array}$

Los 19 casos restantes no reportaron ganglios encontrados en la pieza operatoria. 
Por considerar que el número de casos con diagnóstico histopatológico de ganglios estudiados es muy pequeño, no se hace relación a cifras de supervivencia comparativa similares a las anotadas más arriba por autores extranjeros. López Escobar (12) sobre 90 casos halló ganglios positivos en el $9.7 \%$ de los estados I y en el $21.9 \%$ de los estados II.

\section{Controles:}

Todo médico que haya trabajado en los diferentes hospitales de Bogotá y que se haya interesado por el control de sus pacientes una vez terminado el tratamiento, sabe con amarga experiencia lo difícil que es lograr que toda esa gente que constituye la llamada "Clientela hospitalaria" vuelva periódicamente a los chequeos tantas veces requeridos. La educación de la enferma en este sentido, y sin entrar en consideraciones generales y básicas de educación elemental de nuestro pueblo, es deber primordial del cuerpo médico principalmente en nuestros hospitales de caridad. Pese a los sinceros esfuerzos que se hacen en este sentido por parte de Instituciones como el Instituto Nacional de Cancerología, el porcentaje de pacientes controladas es siempre bajísimo. En nuestro caso particular y con referencia a este trabajo en especial, enviamos cartas a todas las pacientes que según la historia clínica, no habían fallecido, dirigiendo el sobre cerrado al cura párroco como segundo destinatario. En dichas cartas solicitábamos información acerca de la supervivencia y evolución de las enfermas. Solamente recibimos dos de las muchísimas cartas enviadas, y estas dos volvieron a nuestras manos por dirección errada, factor de frecuente ocurrencia dado el estado de la mayoría de las historias clínicas.

La importancia que tienen estos controles puede apreciarse al estudiar la mayoría de las estadísticas extranjeras donde los porcentajes de controles de cinco y diez años son generalmente altos. Es así como el "Reporte Anual" de los resultados del tratamiento del Carcinoma del útero para los años 1945-1954 (10) y sobre un total de 156.253 casos tratados en todo el mundo, da una cifra de $98 \%$ de casos controlados por cinco o más años. En contraste con la gran mayoría de nuestros trabajos donde la falta de controles adecuados impide por definición llegar a conclusiones valederas aplicables directamente a nuestro medio. 


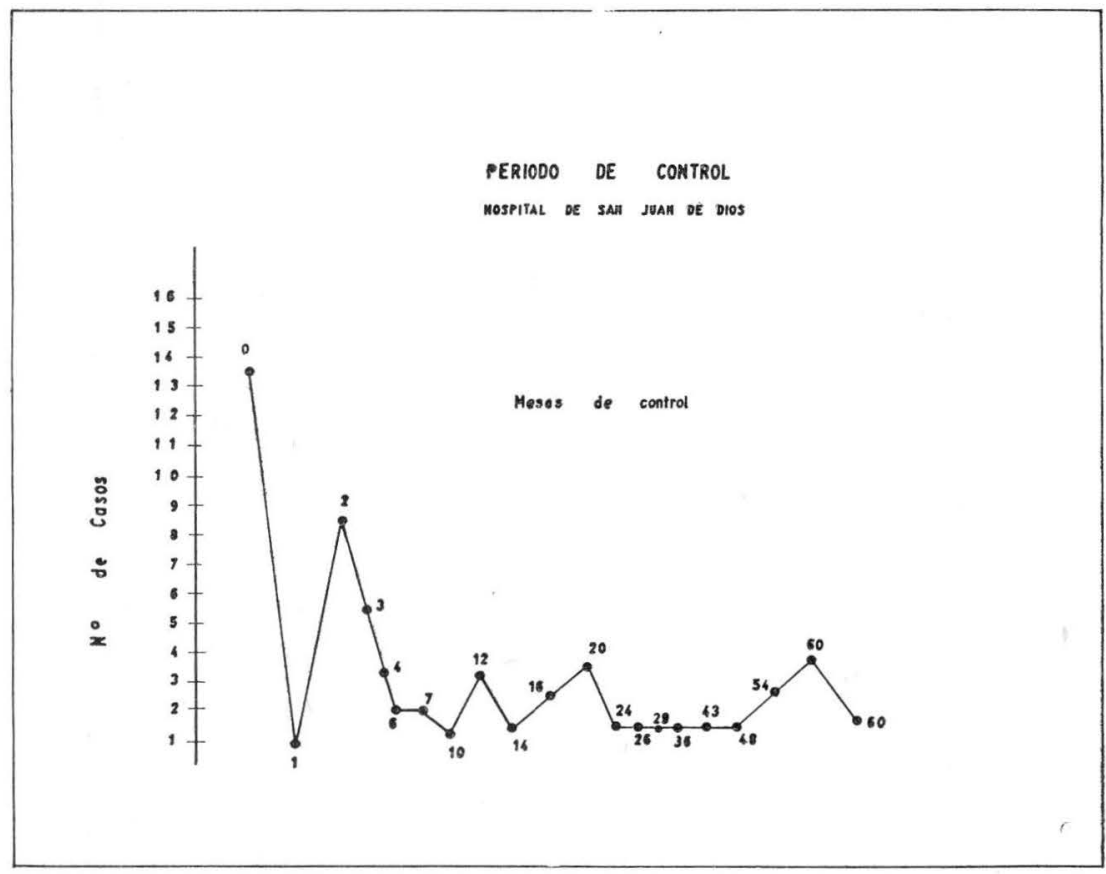

CUADRO No 14

El control de las pacientes operadas en el Hospital de San Juan de Dios de Bogotá ha sido francamente inadecuado como puede observarse en el cuadro $\mathrm{N}^{0} 14$.

El método universalmente aceptado de informar acerca del control post-terapéutico en Carcinoma de cuello uterino se hace por medio del cálculo de las ratas de supervivencia al término de cinco años. Una de las objeciones principales a este método, es la anotada recientemente por Butcher (2) quien pone de presente que con este estricto criterı, solamente una pequeña parte de las pacientes sometidas a tratamiento puede ser usada en los estudios estadísticos. Una rápida ojeada al cuadro anterior nos hace ver claramente la amarga realidad de dicho problema entre nosotros. En su trabajo publicado en Diciembre de 1955 López Escobar encontró un 50\% de los casos perdidos de vista al término de 5 años y presentó un cuadro estadístico usando todas las pacientes controladas por más de un año. Es así como nosotros y con el objeto de presentar cifras estadísticas que puedan ser objetadas y agrupadas posteriormente con series similares en nues- 
tro país, hemos agrupado los controles de aquellas pacientes observadas por un período mínimo postoperatorio de 12 meses seguí método usado por el autor antes mencionado.

\section{CONTROLES}

\begin{tabular}{|l|c|c|c|c|c|c|}
\hline & Sin Dx & I & II & III & IV & Total \\
\hline $\begin{array}{l}\text { Total casos } \\
\text { controlados } \\
12 \text { o más }\end{array}$ & $X$ & 9 & 9 & 1 & 1 & 21 \\
\hline Buen estado & & 6 & 4 & & & \\
\hline Reproducidos & & 3 & 5 & & 11 \\
\hline Muertes P. O. & & & & & & 9 \\
\hline
\end{tabular}

CUADRO No 15

De las cincuenta y cuatro pacientes operadas un año o más, previamente a la terminación de este trabajo, fue posible el controlar por un período mínimo de un año, solamente a 21 pacientes. La evolución de estos casos puede observarse en el cuadro No 15.

En una época más reciente, Harvey R. Butcher (2), ha propuesto un método de informe acerca de controles post-tratamiento en Carcinoma de cuello uterino, el cual se aparta radicalmente del tradicional de supervivencia de cinco años. Las principales ventajas de dicho método son al parecer las de poder aprovechar practicamente la totalidad de las pacientes tratadas en una serie dada, al agruparlas en un cuadro que el autor llama "Tabla de vida". En esta forma y luego de considerar las ratas de mortaliciad, supervivencia específica y número de pacientes en riesgo para sucesivos intervalos de tiempo o control post-tratamiento, se llega así mediante el uso de sencillas operaciones matemáticas a 
salcular la rata acumulativa de supervivencia que permitirá la comparación de resultados entre varios grupos tratados en sitios diferentes y por métodos diversos de una manera mucho más lógica y significativa que con los métodos tradicionales.

Con el objeto de dar a conocer entre nosotros este método de control o informe cancerológico, así como también con el fin de aprovecharnos de sus evidentes ventajas, a continuación hemos agrupado los 58 casos de esta serie según el esquema usado por Butcher en su trabajo original.

\section{TABLA DE VIDA}

\begin{tabular}{|c|c|c|c|c|}
\hline $\begin{array}{l}\text { Intervalo } \\
\text { en meses }\end{array}$ & $\begin{array}{c}\text { № de Pacientes } \\
\text { en riesgos }\end{array}$ & $\begin{array}{l}\text { No de Pacientes } \\
\text { muertos al final } \\
\text { del intervalo }\end{array}$ & $\begin{array}{l}\text { No de Pacientes } \\
\text { vivos al final } \\
\text { del intervalo }\end{array}$ & $\begin{array}{l}\text { Rata acumulativa } \\
\text { de supervivencia }\end{array}$ \\
\hline $0-12$ & 58 & 44 & 14 & 24 \\
\hline $13-24$ & 11 & 0 & 11 & 24 \\
\hline $25-36$ & 8 & 0 & 8 & 11 \\
\hline $37-48$ & 6 & 0 & 6 & 8 \\
\hline $49-60$ & 1 & 0 & 1 & 6 \\
\hline
\end{tabular}

Para la construcción de la "Tabla de vida" es necesario solamente saber en qué época después del tratamiento, las pacientes murieron o fueron perdidas de vista. Las pacientes consideradas "en riesgo" durante un intervalo dado son aquellas que vivieron durante todo el intervalo (12 meses) más aquellas que murieron durante el mismo intervalo. Las enfermas perdidas de vista durante el primer año de intervalo post-tratamiento se cuentan como muertas. La diferencia entre el número de pacientes vivas al final de un intervalo dado y el número de pacientes en ries- 
go durante ei intervalo siguiente representa el número de enfermas perdidas de vista durante este último. La rata de supervivencia para cada intervalo de 12 meses se calcula dividiendo el número de pacientes vivas al final del intervalo por el número en riesgo al comienzo del mismo.

La rata acumulativa de supervivencia se calcula multiplicando la supervivencia específica por la rata acumulativa del intervalo precedente.

\section{CCMENTARIOS}

\section{I) Diagnóstico:}

La demora en el diagnóstico precoz del Ca. de cuello sigue siendo una de las causas más frecuentes de su alta mortalidad. De ahí la insistencia en recalcar que todo consultorio médico debe ser considerado como un centro de detección del cáncer. No debemos olvidar la importancia de la Citología vaginal como método de diagnóstico así como la facilidad con que la muestra puede ser obtenida y enviada al citólogo. Otro tanto podríamos decir del auge que en los últimos tiempos ha tomado la conización cervical no solo como tratamiento en las cervicitis crónicas y los carcinomas intraepiteliales en pacientes jóvenes sino como método diagnóstico en todas aquellas pacientes con cuellos aparentemente sanos y cıtologías positivas para malignidad.

\section{II) Exámenes de Laboratorio:}

Tres tipos de exámenes especiales que hasta hace poco tiempo no se consideraban de importancia han sido incluídos en el servicio de ginecología como de rutina preoperatoria para esta clase de cirugía, a saber: Balance electrolítico, concepto cardiovascular y urografía. Los dos primeros tienen una importancia innegable en la correcta evaluación de la enferma que va a ser sometida a un gran trauma quirúrgico. Por otra parte a la urografía se le concede en la actualidad un gran valor no solo diagnóstico sino también de pronóstico en el Ca. de cuello uterino.

\section{III) Complicaciones Postoperatorias:}

Las fístulas urinarias continúan dando el más alto porcentaje de complicaciones postoperatorias. Entre las más recientes me- 
didas profilácticas que se han propuesto para este problema, vale destacar la de Novak de Yugoeslavia quien preconiza el evitar la denudación circular del uréter dejándolo libre en la cavidad pélvica y la de Symmonds y Pratt quienes aconsejan la succión continua por seis días del espacio retroperitoneal. La segunda de estas técnicas está siendo experimentada por nosotros desde Enero de 1961 y en un futuro esperamos poder informar acerca de su resultado.

\section{IV) Anatomía Patológica:}

Es quizá uno de los puntos sobre los cuales queremos hacer mayor hincapié, pues es curioso ver que aún hoy el Cirujano no se toma la molestia de explicar al Patólogo el origen de la pieza enviada al Laboratoric. No basta con decir "Histerectomía Radical por Ca. de cuello" o "útero y anexos obtenidos por histerectomía total con linfadenectomía", sino que es necesario explicar no solo qué porción vaginal fue resecada, sino el origen de los ganglios, no contentándose con marcar estos últimos derechos e izquierdos, sino que es necesario explicar su origen. (Aórticos-ilíacos-obturadores, etc.) y exigir al Patólogo hacer cortes de todos y cada uno de ellos, así como de la línea de resección.

\section{V) Controles:}

Es indudable que la falta de controles adecuados en las series aquí presentadas igual que en todos los trabajos similares en nuestro medio constituye un factor que demerita en gran parte nuestros esfuerzos por constituír estadísticas nacionales. $\mathrm{Si}$ bien es cierto que básicamente es este un problema de educación general, corresponde directamente al cuerpo médico llevar a cabo esta labor a través de su permanente contacto con las pacientes.

\section{RESUMEN}

$1^{\text {o }}$ - Se revisan cincuenta y ocho casos consecutivos de Carcinoma de cuello uterino tratados quirúrgicamente en el Departamento de Ginecología deì Hospital San Juan de Dios de Bogotá en un período comprendido entre 1955 y 1961. 
$2^{\circ}$ - La mayoría de los casos (44.8\%) estuvieron comprendidos entre los cuarenta y los cincuenta años de edad.

$3^{\circ}$ - El síntoma inicial más frecuente fue el de hemorragia genital, intermenstrual o post-coito presente en el $74 \%$ de los casos.

$4^{\circ}$ - El análisis de las historias, respecto a la demora en el diagnóstico, reveló que en 46 casos fue debida a la paciente misma; entre tanto que en los 12 restantes la demora fue imputable al médico.

$5^{\circ}$ - Se hacen comentarios acerca de los exámenes preoperatorios rutinarios y especiales requeridos en este tipo de Cirugía.

$6^{0}$ - La distribución de los casos aquí presentados según su estado Clínico fue como sigue: Estado I: 24 casos (41.3\%), estado II : 29 casos $(50 \%)$, estado III: 1 caso $(1.7 \%)$, estado IV: 1 caso $(1.7 \%)$, y sin diagnóstico 3 casos $(5.1 \%)$.

$7^{\circ}$ - Se presenta una nueva clasificación de Carcinoma de cuello, basada en los hallazgos quirúrgicos la cual fue descrita inicialmente por Meigs y Brunschwig.

$8^{\circ}$ - La morbilidad operatoria fue del $24 \%$ y la mortalidad general del $3.4 \%$

$9^{9}$ - Las complicaciones post-operatorias más frecuentes fueron de tipo infeccioso (22 casos), fístulas urinarias (14 casos) y complicaciones hemorrágicas (8 casos).

$10^{\circ}$ - De las 54 pacientes operadas hasta julio de 1960, solamente 21 , o sea el $38 \%$ pudieron ser controladas por un período de doce meses. De éstas, 11 se encontraban en buen estado y nueve presentaban carcinoma reproducido.

\section{BIBLIOGRAFIA}

1 BURNS B. C. Jr., EVERETT H. S., BRACK C. B. Value of urologic study in the management of carcinoma of the cervix. Am. J. Obst. a Gynec. 80: 997. Nov. 1960.

2 BUTCHER, HARVEY R. The Reporting of Survival From Cancer. Clin. Obs. \& Gynec. Vol. 4 N: 2 Junio 1961.

3 BRUNSCHWIG A., FRICK H. C Urinary tract fistulas following radical surgical treatment of carcinoma of the cervix. Am. J. Obs. \& Gynec. Vol. 72 № 3 Septiembre 1956. 
4 DIDDLE A. W., KINLAW S. Cervical Carcinoma. Radical hysterectomy and pelvic lymphadenectomy. Am. J. Obs. \& Gynec. Vol. 81 N? 4 Abril 1961.

5 FRICK Hc., TAYLOR. Hc. Jr., GUTTMAN R. J., JACOX H. W., MCKELWAY W. P. A Study of complications in the surgical and radiation therapy of cancer of the cervix. Surg. Gynec. Obst. III: 493 Octubre 1960.

6 HANAFEE W., OTTOMAN R. E., WILK S. P. Roentgen diagnosis of urinary complica tions following radical hysterectomy and pelvic lymph node desection. Radiology Vol. $70 \mathrm{~N}$ : 1 Enero 1958.

7 HORNE H. W. Jr. Carcinoma of the Cervix Uteri. 1926 1948. Obst. Gynec. N. Y. Vol. 9 № 2 Febrero 195\%.

8 JORDAN A. GERMAN. Estudio de $\mathbf{4 5}$ casos de cáncer de cuello uterino tratados quirúrgicamente en el Instituto Nacional de Cancerología. Rev. Col. Obs. \& Ginec. Vol. 6 No 3 Enero. Febrero 1955.

9 KIEKHOFER W.. LEWIS R. S., RICE T. J., CAMPBELL R. E. A resume of carcinoma of the uterine cervix at the University of Wisconsin Hospitals. Obst. Gynec. N. Y. Vol. 14: 559 Noviembre 1959.

10 KOTTMEIR H. L. (Editor) Annual report on the results of treatment in carcinoma of the uterus 1945-1954. Vol. XII, Stockholm 1960.

11 LOCK Fr., GREISS F. C., BLAKE D. D. Stage I carcinoma of the uterine cervix; comparisons of results with variations in treatment. Am. J. Obst. \& Gynec. Vol. 80: 984 Noviembre 1960.

12 LOPEZ E. GUILLERMO. Tratamiento quirúrgico del cáncer del cérvix (parte de una ponencia). Rev. Col. Obst. \& Ginec. Memorias de la segunda convención Colombiana de Obst. \& Ginec. Noviembre-Diciembre 1955.

13 MALLIK BASU. A Study of the ureters following Wertheim's hysterectomy. J. Obstet. \& Ginaec Brit. Emp. Vol 67: 556 Agosto 1960

14 J. V. MEIGS and BRUNSCHWIG A. Proposed clasification for cases of carcinoma of the cervix treated by surgery. Am. J. Obst. \& Gynec. Vol. 64: 413, 1952.

15 MEIGS J. V. Radical hysterectomy with bilateral pelvic lymph-node disection for cancer of the uterine cervix. Clin. Obst. \& Gynec. Vol. 1 N: 4 Diciembre 1958.

16 MEIGS J. V. Cancer of the cervix, an appraisal. Am. J. Obst. \& Gynec. Vol. 72 Ni 3 Septiembre 1956.

17 MEIGS. Am. J. Obst. \& Ginec. Vol. 64: 413, 1952.

18 NOVAK F. Procedure for the reduction of the number of ureterovaginal fistulas after Wertheim's operation. Arrı. J. Obs \& Ginec. Vol. 72 N. 3 Septiembre 1956. cancer of the cervix. Su:g. Gynec. Obstet. 109: 279 Spetiembre 1959.

20 STANDER R. W., RHAMY R. K.. HENDERSON W. P., LANSFORD K. G., PEARCY $M$. The intravenous pyelogram and carcinoma of the cervix. Obst. Gynec. N. $Y$. Vol 17: 26 Enero 1961.

21 SYMMONDS R. E., PRATT J. H. Prevention of fistulas and lymphocysts in radical hysterectomy. Preliminary report of a new technic. Obstet \& Gynec N. Y. Vol. 17: 57 Enero 1961.

22 WELCH J. S., PRATT J. H., SYMMONDS R. E. The Wertheim hysterectomy for squamous cell carcinoma of the uterine cervix. Am. J. Obst. \& Gynec. Vol 81 N: 5 Mayo 1961 